\section{OBESITY}

\section{Is beige the new brown?}

Fat tissue comes in different colors-white, brown and beige. The two latter types express the gene encoding mitochondrial uncoupling protein 1 (Ucp1), allowing them to burn excess fuel. Strategies to increase brown and beige fat in vivo are therefore being intensively explored to combat obesity. A recent report in Nature (doi:10.1038/nature10777) offers new hope in this direction.

Boström et al. found that mice on an exercise regimen or those that transgenically overexpress the peroxisome-proliferatoractivated receptor $\gamma$ coactivator $1 \alpha$ (PGC- $1 \alpha$ ) in the muscle (to mimic exercise) showed Ucp1 upregulation in their subcutaneous fat and, thus, fat 'beiging'. They then took a systems biology approach to identify factors released by the muscle in response to PGC- $1 \alpha$ activity. One of these factors, a shed fragment of fibronectin type III domain containing 5 (FNDC5), which they named irisin, acts as a hormone on subcutaneous white fat cells to induce Ucp1 expression, thus increasing energy expenditure. Genetic overexpression of FNDC5 modestly raised serum concentrations of irisin and reduced body weight in obese mice while improving their insulin sensitivity.

The team also showed that irisin plasma concentrations are higher in people who exercise, suggesting a mechanism by which exercise improves metabolism.

One aspect missing from the report is the identification of the cell-surface receptor for irisin. Once identified, it is possible that small chemical compounds could be developed that target this receptor, thus providing a drug platform to turn white fat beige and reduce obesity in the clinic. $-R L$

\section{INFLAMMATION}

\section{Burning up the brain}

Several hypotheses have been put forth to describe how production of the cytokine interleukin $1 \beta$ (IL-1 $\beta$ ) in the periphery induces fever, including activation of neurons and microglia, direct stimulation of hypothalamic neurons and peripheral activation of the vagus nerve.

Now, Markus Schwaninger and his colleagues (J. Exp. Med. 208, 2615-2623) report that brain endothelial cells are the target of IL-1 $\beta$ in fever. In vitro IL- $1 \beta$ activates the MAP kinase kinase kinase TAK1. This induces cyclooxygenase- 2 and results in prostaglandin E2 production in brain endothelial cells, leading to fever and lethargy. Conditional deletion of TAK 1 specifically in brain endothelial

ANTIMICROBIALS

\title{
Mycobacterial asymmetry
}

A new study provides insights

into how variable antibiotic

susceptibility may arise in

mycobacterium populations,

which may have implications for

our understanding of how to best

apply antibiotic treatments to

eliminate infection in individuals

with tuberculosis.

Aldridge et al. (Science 335,

100-104) investigated the

growth and division of single

Mycobacterium smegmatis

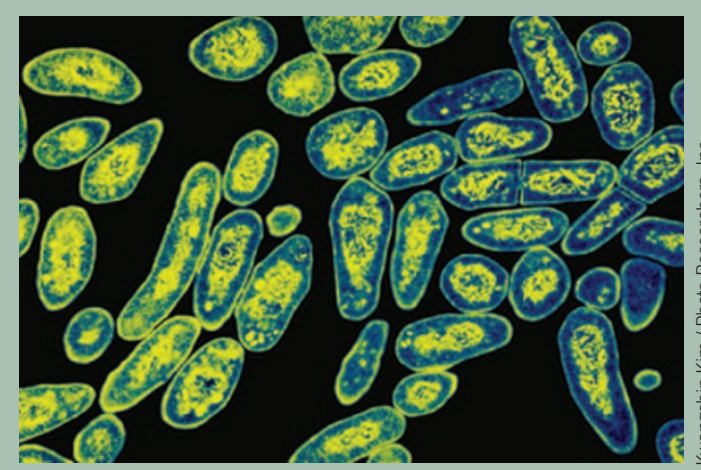

and Mycobacterium tuberculosis cells and found that, compared to Escherichia coli, the mycobacterial cells had more variable elongation rates. $E$. coli cells divide symmetrically and elongate along the lateral cell body; in contrast, mycobacterial cells divide asymmetrically and elongate in a unipolar manner from the growth pole. The authors found that these characteristics result in two different daughter cells after M. smegmatis division: an 'accelerator cell', which inherits the old growth pole and grows more rapidly, and an 'alternator cell', which must assemble a new growth pole and therefore grows more slowly than the accelerator cell.

Antibiotics target bacterial processes crucial to growth and division, and Aldridge et al. found that, consistent with their faster growth rate, accelerator cells were more sensitive than alternator cells to antibiotics that inhibit cell-wall synthesis. Future studies will be necessary to determine whether these variations observed in $M$. smegmatis populations also apply to $M$. tuberculosis and whether there are consequences for antibiotic resistance of mycobacteria in vivo. $-M S$

cells in mice reduced the IL-1 $\beta$-induced fever response and lethargy. TAK 1 ablation in neurons, astrocytes and oligodendrocytes did not alter this response, ruling out a contribution by these cell types.

In addition to fever and lethargy, the IL-1 $\beta-$ induced sickness response also includes anorexia, weight loss and activation of the hypothalamic-pituitary axis in mice. However, these latter characteristics were not affected by TAK 1 deletion in brain endothelial cells, suggesting that IL-1 $\beta$ may affect different elements of the sickness response by acting on distinct cell populations. - KDS

\section{BRAIN}

\section{Anxious about your meal?}

The sirtuin SIRT1 functions in the brain to regulate several metabolic processes, as well as affecting memory and learning. A new study in mice shows that this deacetylase can also influence anxiety and behavior (Ce/l 147, 1459-1472).

Sergiy Libert and his colleagues showed that brain-specific knockout of SIRT1 decreased anxiety, promoted exploratory drive and resulted in increased amounts of the neurotransmitter serotonin in the brain. SIRT1-overexpressing mice had the opposite phenotype, which could be corrected using inhibitors of monoamine oxidase A (MAO-A) — an enzyme that metabolizes serotonin. The authors also characterized how SIRT1 can regulate the amount of serotoninSIRT1 boosts MAOA transcription by deacetylating the transcription factor nescient helix loop helix 2 (NHLH2) on Lys49, thus increasing its activity on the MAOA promoter and decreasing the amount of serotonin in the brain.

Large-scale genetic analyses revealed an association between certain SIRT1 human polymorphisms and an increased risk of anxiety, social and panic disorders. Furthermore, two of the most common SIRT1 variants found in people at risk of developing anxiety disorders enhanced SIRT1 activity and its ability to activate MAO-A in vitro.

SIRT1-mediated regulation of anxiety probably has a role in adaptation to environmental variations, such as food abundance, which suppresses SIRT1. The results from this study suggest that targeting SIRT1 in the brain may also be promising for the treatment of human anxiety. $-C P$

Written by Kevin Da Silva, Randy Levinson, Carolina Pola and Meera Swami. 